\title{
Robust Shape Normalization Based on Implicit Representations
}

\author{
Tingting Jiang \\ LEAR group, INRIA Grenoble, France \\ tjiang@inrialpes.fr
}

\author{
Carlo Tomasi \\ Department of Computer Science \\ Duke University, USA \\ tomasi@cs.duke.edu
}

\begin{abstract}
We introduce a new shape normalization method based on implicit shape representations. The proposed method is robust with respect to deformations and invariant to similarity transformations (translation, isotropic scaling and rotation). The new method has been tested and compared to the classical shape normalization method and previous work in terms of aligning groups of shapes with deformations.
\end{abstract}

\section{Introduction and Related Work}

One of the most important goals for computer vision is to recognize objects automatically. Human visual system achieves this goal efficiently based on various input information, such as shape, color and texture. Among these inputs, shape is one of the most useful clues because color and texture can change easily within the same class of objects, such as cars or pedestrians, while shape is relatively static. However, the shapes of objects within the same class can also vary to a certain extent. For example, the object could be viewed from different perspectives or it could be nonrigid. Therefore, shape normalization becomes a very important step in building shape models.

In general, shape normalization is performed to make shape representations invariant under a given a class of transformations. Therefore, one can classify existing shape normalization methods according to two aspects: (i) shape representation, and (ii) transformations. In terms of representation, different approaches have been proposed, including landmarks like the Procrustes analysis [3], parametric curves or surfaces such as splines [2], or implicit functions such as the distance transforms. In terms of transformations, usually people consider similarity transformations which are translation, isotropic scaling and rotation. Therefore, the specific goal of shape normalization is to find the center, the scale factor and the orientation of the shape to make the shape model invariant w.r.t. the three transformations.

Traditionally, the center of the shape is defined as the geometric center, the shape size is normalized to unit area, and the orientation of the shape is defined as the angle of the axis of the least moment of inertia $[4,5]$. One difficulty is how to reduce the sensitivity of the shape model w.r.t. shape deformations. Based on the traditional method, [1] proposed a robust estimation method for shape orientation.
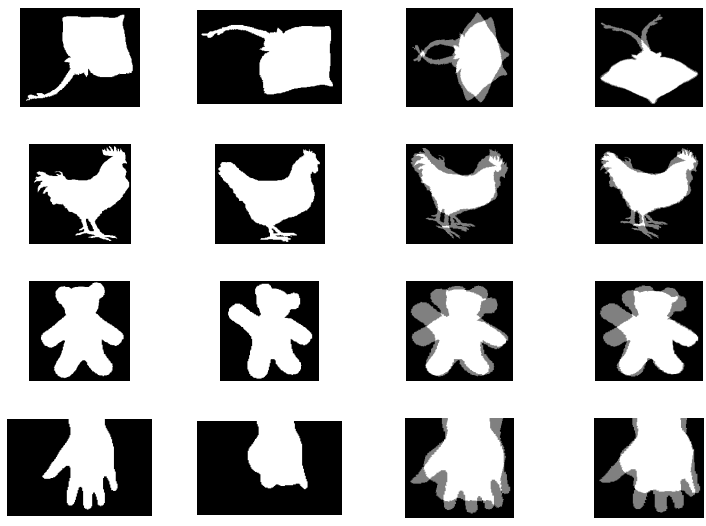

Figure 1. Comparison of the alignments of 4 pairs of shapes (Column 1 and 2) normalized by the traditional method ( Column 3) and the new method ( Column 4).

In this work, we are motivated by some classes of shapes, such as the silhouettes of animals (Fig. 1). Within each of these classes, shapes are composed of both non-articulated parts (main body) and articulated parts (arms, legs, heads, tails, etc.). Observing that nonarticulated parts are usually "fat" while the articulated parts are often "thin", we introduce a new robust shape normalization method based on implicit representations of shapes. The central idea is to use a weight function to 


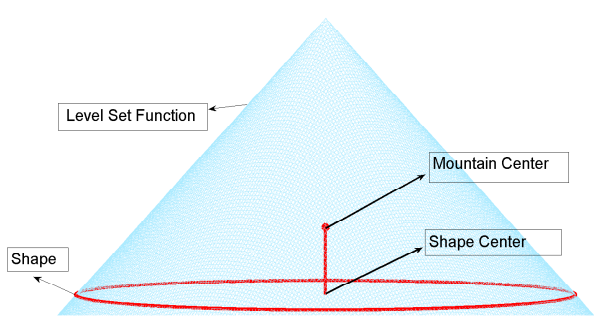

Figure 2. The projection of the mountain center is the shape center $(\lambda=1)$.

emphasize the influence of the fat part (points far away from the boundary) while reducing that of the thin part (points close to the boundary).

The novelty of our approach stems from the way we define the shape center and scaling factor. Unlike in prior work, we seek to make both the shape center and scale robust w.r.t. deformations besides robust shape orientation. In principal, our method can be extended to normalize shapes in higher dimensions because of the nature of implicit representations. Experiments of normalization of different shape classes are presented with comparison to previous work [1]. The results demonstrate that our approach offers a more robust and general solution to shape normalization.

\section{Implicit Shape Representations}

Given a closed planar curve $\mathcal{C}$ which defines a shape $S$, we define a signed distance function $\phi$ as follows:

$$
\phi(x, y)= \begin{cases}d((x, y), \mathcal{C}) & \text { if }(x, y) \text { inside } \mathcal{C} \\ -d((x, y), \mathcal{C}) & \text { if }(x, y) \text { outside } \mathcal{C} \\ 0 & \text { if }(x, y) \in \mathcal{C}\end{cases}
$$

where $d((x, y), \mathcal{C})$ is the minimum Euclidean distance between the point $(x, y)$ and the curve $\mathcal{C}$. Therefore, $\phi(x, y)$ embeds $\mathcal{C}$ as its zero-level set and has unitnorm gradient. This implicit representation [6] can also describe multi-component shapes and has been widely used in image analysis.

\section{Robust Shape Normalization}

Based on implicit representations, we propose a new shape normalization method to reduce the sensitivity w.r.t. the shape deformations. The central idea is to

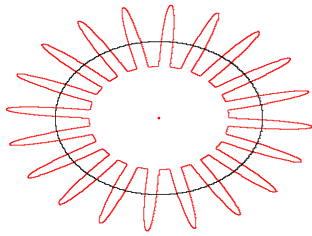

(a)

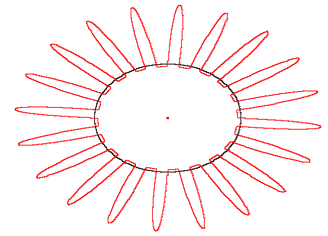

(b)
Figure 3. The aligment of a circle and a star shape normalized by (a) the area and (b) the mass $V(\lambda=1)$.

design a density function $\rho(x, y)$ for a shape based on its implicit representation $\phi$ as

$$
\rho(x, y)=\phi^{\lambda}(x, y)(\lambda=1,2, \ldots)
$$

and then redefine the center, scale factor and orientation of the shape as follows. The parameter $\lambda$ controls the degree of robustness. The larger $\lambda$ is, the less sensitive the shape normalization is w.r.t. shape deformations.

Center. The center of shape is defined as the center of mass with the density function $\rho$, i.e.,

$$
(\hat{x}, \hat{y})=\frac{\iint_{\phi(x, y) \geq 0}(x, y) \rho(x, y) \mathrm{d} x \mathrm{~d} y}{\iint_{\phi(x, y) \geq 0} \rho(x, y) \mathrm{d} x \mathrm{~d} y} .
$$

It can be viewed as the weighted average of shape points and the weight is proportional to $\rho(x, y)$.

For a 2D circle, when $\lambda=1$, the corresponding density function $\rho(x, y)$ looks like a mountain in the 3D space (Fig. 2). The projection of the mountain center on the plane coincides with the newly defined center. The mass of the mountain is

$$
V=\iint_{\phi(x, y) \geq 0} \rho(x, y) \mathrm{d} x \mathrm{~d} y .
$$

Because the contribution of each shape point is weighted by $\rho(x, y)$, computing the weighted center of the shape points is equal to computing the projection of the center of the mountain (Fig. 2).

Scale Factor. To normalize the shape size, we define a scale factor as $\alpha=\sqrt[\lambda+2]{V}$, instead of the square root of the shape area. For higher dimensions $D \geq 3$, the scale factor can be generalized as $\alpha=\sqrt[\lambda+D]{V}$. Fig. 3 shows the difference when using different definitions of the scale factor. A circle and a star shape are normalized by the area ( Fig. 3(a)), and by the mass ( Fig. 3(b)) respectively. It is easy to see that the latter alignment is more resistant to the branches. 

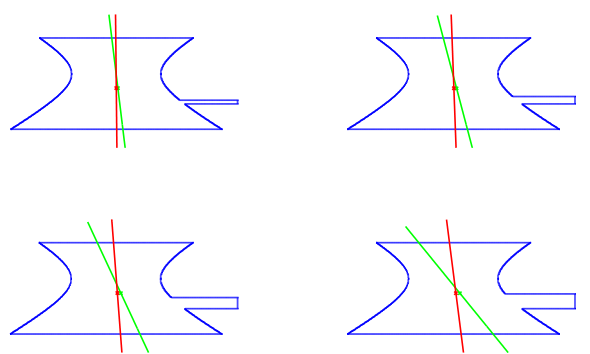

Figure 4. Compare the orientations by traditional method (green) and our new method (red) with $\lambda=1$.

Orientation. In order to make the orientation robust, we define the axis to be the line which minimizes the inertia with the density function $\rho$ as:

$$
I_{2}=\iint_{\phi(x, y) \geq 0} r^{2}(x, y) \rho(x, y) \mathrm{d} x \mathrm{~d} y .
$$

where $r(x, y)$ denotes the shortest distance from point $(x, y)$ to the line. Compared to the traditional approach of minimizing inertia $[4,5]$, which is

$$
I_{1}=\iint_{\phi(x, y) \geq 0} r^{2}(x, y) \mathrm{d} x \mathrm{~d} y,
$$

minimizing $I_{2}$ will be less affected by the change of $r(x, y)$ for points closer to the boundary because their values of $\rho(x, y)$ are smaller. Therefore, the orientation is more robust to shape deformations than the traditional method (Fig. 4). It is easy to show that the axis of minimizing $I_{2}$ passes through the center in Eqn. (2) and its angle $\theta$ satisfies $\tan 2 \theta=\frac{B}{A-C}$ unless $B=0$ and $C=A$ where

$$
\begin{aligned}
A & =\iint_{\phi(x, y) \geq 0}(x-\hat{x})^{2} \rho(x, y) \mathrm{d} x \mathrm{~d} y, \\
B & =2 \iint_{\phi(x, y) \geq 0}(x-\hat{x})(y-\hat{y}) \rho(x, y) \mathrm{d} x \mathrm{~d} y, \\
C & =\iint_{\phi(x, y) \geq 0}(y-\hat{y})^{2} \rho(x, y) \mathrm{d} x \mathrm{~d} y .
\end{aligned}
$$

From the above definitions about center, scale and orientation, we can see that the traditional shape normalization approach is one special case when $\lambda=0$.

Direction Ambiguity. With the way we have defined the axis of a shape so far, we would get the same result if

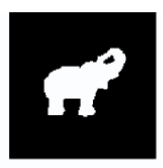

$1 \mathrm{a}$

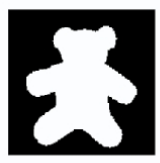

$1 \mathrm{e}$

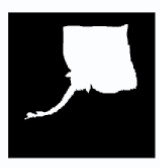

1i

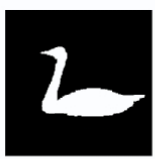

$1 \mathrm{~m}$

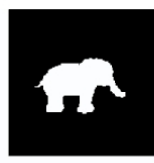

$1 \mathrm{~b}$

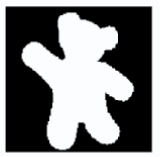

1f

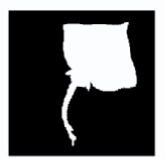

$1 \mathrm{j}$

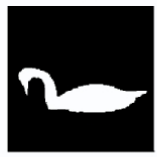

1n

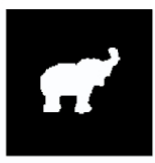

$1 \mathrm{c}$

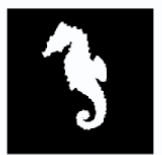

$1 \mathrm{~g}$

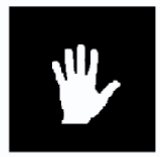

$1 \mathrm{k}$

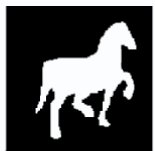

10

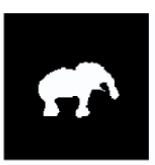

$1 d$

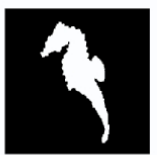

$1 \mathrm{~h}$

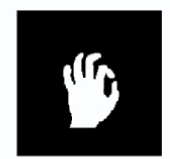

11

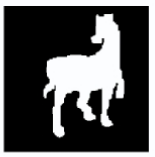

$1 \mathrm{p}$
Figure 5. Eight pairs of shapes used for testing the robustness of the localization and orientation estimation [1].

we rotated the shape 180 degrees and then recomputed the axis. One solution to resolve the ambiguity is to let the orientation of the shape point towards the half plane that contains the region of the shape with larger weighted area [1]. Here we choose the direction which is closer to the "average" direction of gradient vectors of the implicit level set function, which is defined as the weighted sum of the normalized gradient vectors of $\phi$ :

$$
\vec{v}=\iint_{\phi(x, y) \geq 0} \frac{\nabla \phi(x, y) \rho(x, y)}{\|\nabla \phi(x, y)\|} \mathrm{d} x \mathrm{~d} y .
$$

\section{Experimental Results}

Several experiments have been carried out to test the effectiveness of the proposed method.

The first one compares the shape alignments by the traditional method and the new method. In Fig. 1, there are four pairs of shapes normalized and aligned respectively by the traditional method (third column) and the new method (last column). Each row corresponds to a group of shapes. The first two columns are the given four pairs of shapes. The white area denotes the overlap and the gray denotes the difference between the aligned 


\begin{tabular}{|c|c|c|c|c|}
\hline & $\begin{array}{c}\Delta \theta_{1} \\
(\text { degree) }\end{array}$ & $\begin{array}{c}\Delta \theta_{2} \\
(\text { degree) }\end{array}$ & $\begin{array}{c}\Delta C_{1} \\
\text { (pixel) }\end{array}$ & $\begin{array}{c}\Delta C_{2} \\
\text { (pixel) }\end{array}$ \\
\hline $1 \mathrm{a} / 1 \mathrm{~b}$ & 1.33 & 0.53 & 3 & 0 \\
\hline $1 \mathrm{c} / 1 \mathrm{~d}$ & 0.28 & 0.90 & 2 & 4 \\
\hline $1 \mathrm{e} / 1 \mathrm{f}$ & 3.71 & 0.47 & 3 & 0 \\
\hline $1 \mathrm{~g} / 1 \mathrm{~h}$ & 0.07 & 0.55 & 2 & 0 \\
\hline $1 \mathrm{i} / 1 \mathrm{j}$ & 0.00 & 0.10 & 0 & 0 \\
\hline $1 \mathrm{k} / 1 \mathrm{l}$ & 0.11 & 0.96 & 0 & 0 \\
\hline $1 \mathrm{~m} / 1 \mathrm{n}$ & 0.00 & 0.63 & 2 & 0 \\
\hline $1 \mathrm{o} / 1 \mathrm{p}$ & 4.53 & 3.69 & 5 & 4 \\
\hline
\end{tabular}

Table 1. Compare robustness of localization and orientation estimation of shapes in Fig. 5 between our method and [1].

normalized shapes. We can see that the new shape normalization method performs better w.r.t. the deformations within the same class of shapes, especially for the first and the last row. In the last row, the new method aligns the fist with the full hand better than the traditional method because the latter normalizes the size of both shapes to unit area and results that the fist looks larger than the full hand in the alignment.

The second experiment compares the robustness of localization and orientation estimation between the proposed method and [1]. Fig. 5 shows 8 pairs of shapes with slight deformations. The parameter used is $\lambda=5$ and image size is $128 \times 128$. Two parameters $\Delta \theta$ and $\Delta C$ are computed. $\Delta \theta$ denotes the difference between the orientation estimations of two similar shapes. $\Delta C$ denotes the difference between the shape center estimations of two shapes, using the city-block distance. In Table $1, \Delta \theta_{1}$ and $\Delta C_{1}$ are results from [1], while $\Delta \theta_{2}$ and $\Delta C_{2}$ are our results. Comparison of $\Delta C_{1}$ and $\Delta C_{2}$ suggests that the shape center estimation by our method is less sensitive to deformations in general. As for the orientation, it is hard to say which one is better in general. Notice that in this experiment scaling factor is not considered. In [1], the size adjustment depends on a predefined parameter and sometimes can crop the shapes. However, this won't happen in our approach.

The last experiment is to show the influence of $\lambda$. With larger $\lambda$, the normalization is more robust to the deformations. In Fig. 6, the alignment of the two elephant shapes (the first row) gets better as $\lambda$ increases.

\section{Conclusions and Future Work}

In this paper, we proposed a new shape normalization method which is invariant to similarity transformations and robust to shape deformations. The close-form

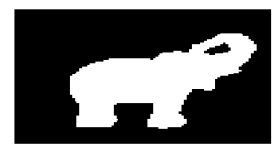

$\lambda=1$

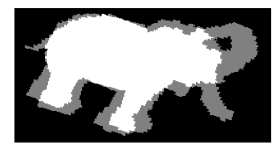

$\lambda=3$

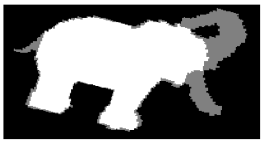

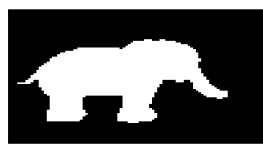

$\lambda=2$

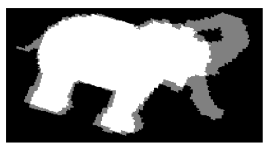

$\lambda=4$

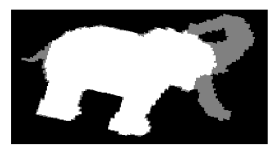

Figure 6. Alignments of elephant shapes by the new method with increasing $\lambda=$ $1,2,3,4$. The first row shows the given pair of elephant shapes.

solution provides a simple and efficient way to estimate the shape center, scale factor and orientation. Compared to previous work, our primary contribution is that the proposed method can reduce not only the sensitivity of the orientation, but also the sensitivity of center and scale factor to the deformations. The experiments have shown that this method performs well for aligning shapes with deformations.

There are several limitations which we intend to overcome in future. First, the current shape orientation method does not work for $n$-fold $(n>2)$ rotationally symmetric shapes as the traditional method. Second, the solution for the direction ambiguity is not stable for shapes which are nearly symmetric. We hope to rationalize the selection of the orientation. Although extension to higher dimensions $(D \geq 3)$ is conceptually straight-forward, we need to experiment with this generalization to ensure that it is both feasible and useful.

\section{References}

[1] J. Cortadellas, J. Amat, and F. de la Torre. Robust normalization of silhouettes for recognition applications. Pattern Recognition Letters, 25(5):591-601, 2004.

[2] C. De Boor. A Practical Introduction to Splines. Springer, New York, NY, 2001.

[3] I. L. Dryden and K. V. Mardia. Statistical Shape Analysis. Wiley, Chichester, 1997.

[4] B. K. P. Horn. Robot Vision. MIT Press, Cambridge, Massachusetts, 1986.

[5] R. C. Jain, R. Kasturi, and B. G. Schunck. Machine Vision. McGraw-Hill, 1995.

[6] N. Paragios, M. Taron, X. Huang, M. Rousson, and D. Metaxas. On the representation of shapes using implicit functions. Statistics and Analysis of Shapes, 2006. 